\title{
Cardiac mediated cerebral blood flow changes in chronic hydrocephalus
}

\author{
Mark G Luciano*1, Stephen M Dombrowski ${ }^{1}$, Soren Schenk ${ }^{2}$, \\ Anna Leichliter ${ }^{1}$, Zack Leibson ${ }^{1}$ and Kiyotaka Fukamachi ${ }^{2}$
}

\begin{abstract}
Address: ${ }^{1}$ Department of Neurological Surgery, S-80 Pediatric and Congenital Neurological Surgery, The Cleveland Clinic Foundation, 9500 Euclid Avenue, Cleveland, OH 44195, USA and 2Department of Biomedical Engineering, ND-20, Lerner Research Institute, The Cleveland Clinic Foundation, 9500 Euclid Avenue, Cleveland, OH 44195, USA

Email: Mark G Luciano* - lucianm@ccf.org

* Corresponding author
\end{abstract}

from 50th Annual Meeting of the Society for Research into Hydrocephalus and Spina Bifida

Cambridge, UK. 30 August - 2 September 2006

Published: 21 December 2006

Cerebrospinal Fluid Research 2006, 3(SuppI I):S28 doi:I0.1186/1743-8454-3-SI-S28

(C) 2006 Luciano et al; licensee BioMed Central Ltd.

\section{Background}

Increased intracranial pressure (ICP), vascular compression as the result of enlarged cerebrospinal fluid (CSF) spaces, or impaired metabolic activity may be responsible for decreased cerebral blood flow (CBF) seen in hydrocephalus. Little attention has been given to the relationship between cardiac function and systemic blood flow in chronic hydrocephalus $(\mathrm{CH})$.

\section{Materials and methods}

Using an experimental model of chronic obstructive hydrocephalus developed in our laboratory, we investigated the relationship between hydrocephalus duration and severity and cardiac output (CO), cerebral blood flow (CBF), myocardial tissue perfusion (MTP), and peripheral blood flow (PBF). Blood flow measures were obtained via microsphere injection method under controlled hemodynamic conditions in experimental $\mathrm{CH}(\mathrm{n}=23)$ and surgical control $(\mathrm{n}=8)$ canines at baseline and 2, 4, 8, 12, and 16 weeks. CO measures were made using the Swan-Ganz thermodilution method. Intracranial compliance (ICC) via CSF bolus removal and infusion, and oxygen delivery in CSF and prefrontal cortex (PFC) were also investigated.

\section{Results}

We observed an initial surgical effect relating to $30 \% \mathrm{CO}$ reduction and $\sim 50 \%$ decrease in $\mathrm{CBF}, \mathrm{MTP}$, and $\mathrm{PBF}$ in both groups 2 weeks post-operatively that recovered in control animals but continued to decline further in $\mathrm{CH}$ animals at 16 weeks. CBF, which was positively correlated with CO ( $\mathrm{p}=0.028)$, showed no significant relationship with either CSF volume or pressure. Decreased CBF correlated with oxygen deprivation in PFC $(\mathrm{p}=0.006)$. CO was inversely related with ventriculomegaly $(\mathrm{p}=0.019)$, but did not correlate with ICP. Decreased CO corresponded to increased ICC as measured via CSF infusion $(p=0.04)$.

\section{Conclusion}

Our results suggest that $\mathrm{CH}$ may have more of an influence on $\mathrm{CO}$ and $\mathrm{CBF}$ in the chronic stage than in the early condition, which was dominated by surgical effect. The cause of this late deterioration of cardiac function in hydrocephalus is uncertain, but may reflect cardiac regulation secondary to physiological response or brain injury. The relationship between cardiac function and cerebral blood flow should be considered in the pathophysiology and clinical treatment of chronic hydrocephalus. 